Proceedings

\title{
Simulations of Energy Losses in the Bulk Nuclear Medium Using Hydrodynamics on the Graphics Cards (GPU) ${ }^{\dagger}$
}

\author{
Marcin Słodkowski ${ }^{1}$ * (D), Patryk Gawryszewski ${ }^{1}$, Patryk Marcinkowski ${ }^{1}$, Dominik Setniewski ${ }^{1}$ \\ and Joanna Porter-Sobieraj ${ }^{2}$ \\ 1 Faculty of Physics, Warsaw University of Technology, 75, Koszykowa str., 00-662 Warsaw, Poland; \\ patgaw09318@gmail.com (P.G.); piro.plock@gmail.com (P.M.); 274434@pw.edu.pl (D.S.) \\ 2 Faculty of Mathematics and Information Science, Warsaw University of Technology, 75, Koszykowa str., \\ 00-662 Warsaw, Poland; J.Porter@mini.pw.edu.pl \\ * Correspondence: m.slodkowski@if.pw.edu.pl \\ + Presented at Hot Quarks 2018 - Workshop for Young Scientists on the Physics of Ultrarelativistic \\ Nucleus-Nucleus Collisions, Texel, The Netherlands, 7-14 September 2018.
}

Published: 15 April 2019

check for updates

\begin{abstract}
We are developing a software for energy loss simulation which is affected by jets in the nuclear matter described by relativistic hydrodynamics. Our program uses a Cartesian coordinate system in order to provide high spatial resolution for the analysis of jets propagation in nuclear matter. In this work, we use 7th order WENO numerical algorithm which is resistant to numerical oscillations and diffusions. For simulating energy losses in the bulk nuclear medium, we develop efficient hydrodynamic simulation program for parallel computing using Graphics Processing Unit (GPU) and Compute Unified Device Architecture (CUDA). It allows us to prepare event-by-event simulations in high computing precision in order to study jet modifications in the medium and event-by-event simulations of fluctuating initial conditions. In our simulation, we start the hydrodynamic simulation from generation initial condition based on the UrQMD model in order to simulate comparable nucleus-nucleus interaction in the RHIC and LHC energies. The main part of this simulation is the computation of hydrodynamic system evolution. We present obtained energy density distributions which can be compared to experimental results.
\end{abstract}

Keywords: heavy ion collisions; relativistic hydrodynamic; simulations of energy loses in medium; numerical algorithms; WENO; parallel computing; CUDA/GPU

\section{Introduction}

Our study is a part of the investigation of Strongly Interacting Matter (SIM). Heavy nucleus-nucleus interactions are under considerations at SPS, RHIC and LHC experimental facilities. Experimental data together with numerical simulations may give complementary information to extract properties of SIM. One of the open questions in this study is understanding the origin of momenta anisotropy of produced particles during nucleus-nucleus collisions. The source of non-isotropic momentum vectors of the produced particles can be affected by flow and non-flow effects during heavy ion collisions. In order to distinguish those two physical groups of effects, we study signal of bulk/collective properties of jet-medium interactions and initial state fluctuation. By studying jet-medium interaction and jet-induced flow, it is possible to extract information of properties of the Quark-Gluon Plasma (QGP). Fluctuation 
coming from the early non-equilibrium stage can be affected by the dynamic of the nuclear medium. Furthermore, higher Fourier harmonics indicate flow anisotropy and odd Fourier coefficients signal fluctuations in the pre-equilibrium phase. Relativistic hydrodynamics event-by-event simulations are needed in order to study the contribution of initial state fluctuations. Thus the very efficient numeric implementation of the hydrodynamic equations is necessary on the Graphics Cards (GPU) using Compute Unified Device Architecture (CUDA).

\section{Applied Methods}

We are developing hydrodynamic code which is able to perform a simulation of energy losses in bulk nuclear medium which is affected by jets using CUDA architecture and GPU processors. In order to perform the simulation of jets dynamics and jets modification with sufficient accuracy, we assume a high spatial resolution of the computational grid up to $200^{3}$ using the Cartesian coordinate system. Therefore these conditions need a lot of computing power. Moreover, simulations have to be fast enough to obtain reasonable statistics in the event-by-event simulation for flow fluctuations analysis. The main component solves the set of partial hydrodynamic equations base on universal hyperbolic equations of the conservation laws:

$$
\frac{\partial U}{\partial t}+\nabla \cdot F(U)=S^{v}(\vec{r})
$$

$U$ - universal vector conserved hydrodynamic quantities such as energy, momenta, net charge densities, their $F(U)$ flux vector and $S^{v}(\vec{r})$ sources term of modification in the hydrodynamics quantities. We applied a numerical scheme using finite differences methods: the standard 3th order Runge-Kutta methods for time integrations and the 7th order WENO algorithm [1] on GPU processor using CUDA framework. The WENO is a self-balanced numerical algorithm which is resistant to numerical oscillations and diffusion and gives a very good performance. For completeness of description, there is a need to define the Equations of State (EoS) as an input of our program which contains information about the physics of the simulation. For A+A interaction we use ultrarelativistic EoS type for particles with zero rest mass: $p=c_{s}^{2} e$ where $c_{s}^{2}=\frac{1}{3}$. Mechanism of $d E / d x$ is responsible for modelling interaction between the jet and the plasma and expressed by the following term:

$$
\left(-\frac{\mathrm{d} E}{\mathrm{~d} x}\right)=\kappa_{\text {rad }} \frac{C_{R}}{C_{F}} T^{3} x+\kappa_{\text {coll }} \frac{C_{R}}{C_{F}} T^{2}
$$

The energy loss $d E / d x$ algorithm includes gluon radiation and collision of partons in the dense bulk matter. The coefficients ( $\left.\kappa_{\text {rad }}, \kappa_{\text {coll }}, C_{R}, C_{F}\right)$ depend on jet flavor (quark or gluon), its energy and $T$ parameter is local temperature [2]. We have taken in these simulations that $\kappa_{\text {rad }}=4, \kappa_{\text {coll }}=2.5, C_{R} / C_{F}=1$. Numeric algorithms for integrating partial differential equations of relativistic hydrodynamic and energy loss $d E / d x$ mechanisms were implemented on GPU using CUDA framework. Procedures of numerical algorithms were directly executed on the graphics processor. Operations on the cells of the numerical grid are performed in the parallel at the same time avoiding the usage of traditional programming loops.

\section{Results}

The implementation of 7th order WENO numerical algorithm has been successfully examined using three analytical solutions: the SOD shock tube, the Hubble-like expansion, an ellipsoidal flow described in details in Ref. [3]. Our performance tests show large speed-up of our parallel thread simulations on GPU in comparing single thread simulation on classical CPU (at least 2 orders of magnitude). 

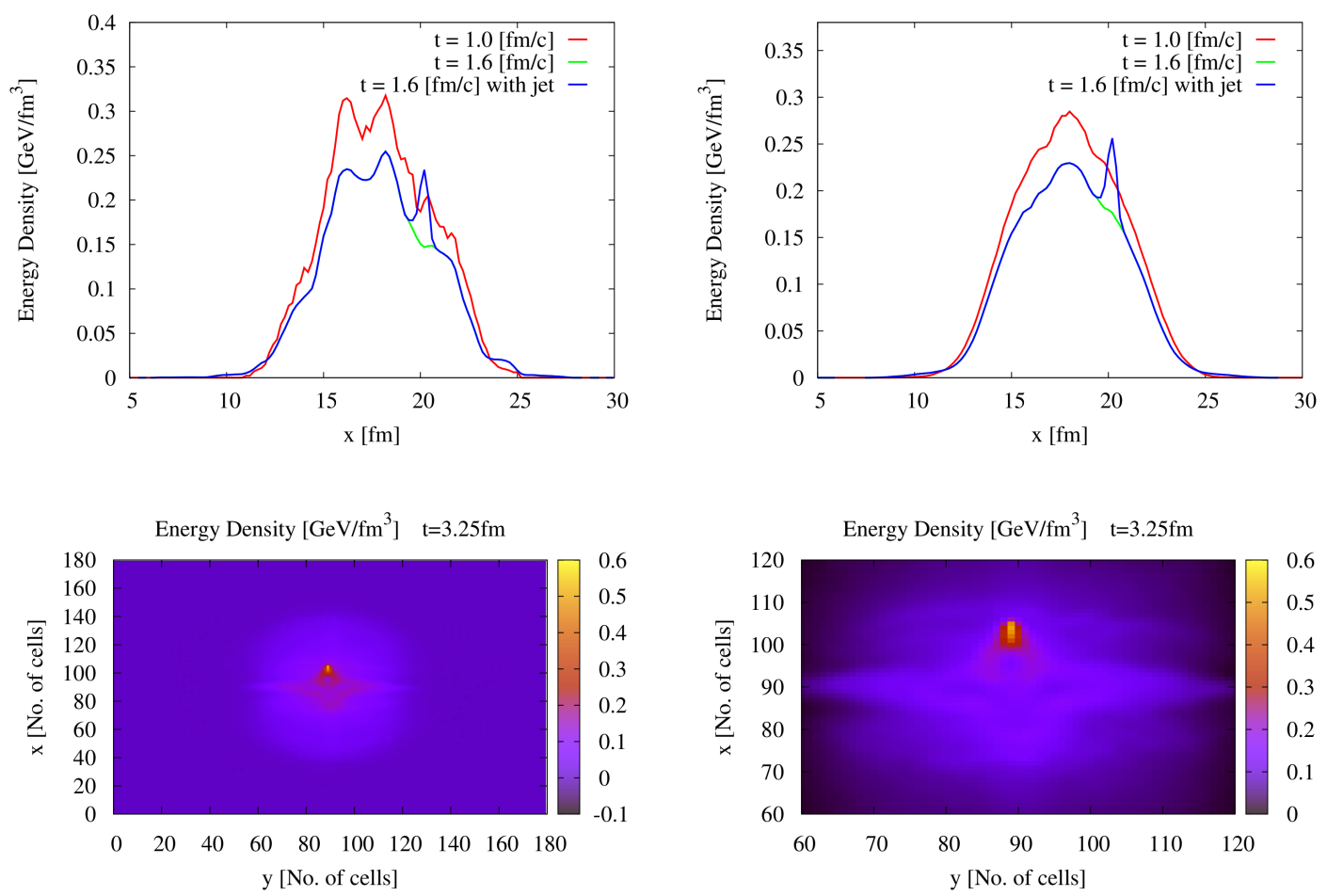

Figure 1. Energy density projection in $1 \mathrm{D}(y=z=0)$ of $\mathrm{Au}+\mathrm{Au}$ at $200 \mathrm{GeV} / \mathrm{c}$ UrQMD initial conditions - red lines. Hydrodynamic simulations with jet - blue lines, without jet - green lines (top panels). Initial condition from UrQMD averaging over 10 events (top left panel) and 500 events (top right panel). Energy density cross-section of $\mathrm{Au}+\mathrm{Au}$ at $200 \mathrm{GeV} / \mathrm{c}$ in $2 \mathrm{D}$ ( $x y$ plane with $z=0$ and cell size is $\Delta x=\Delta y=\Delta z=$ $0.166[\mathrm{fm}]$ ) (bottom left panel). Energy density profile zoom $\times 3$ (bottom right panel).

Our nucleus-nucleus interaction simulations begin from the generation of initial distributions coming from UrQMD model [4]. In heavy ions collisions, it is difficult to describe the pre-equilibrium stage due to deficiencies in the theory. It is known that in the early stage there are fluctuations which are caused by non-equilibrium processes at the parton level, therefore, certain approximations are introduced. Initial state fluctuations can be obtained using Glauber Monte-Carlo, models like UrQMD, other string models and another approach. The UrQMD is used to simulate only the early phase of the collision. Time of the UrQMD simulations was set to $1 \mathrm{fm}$. The result of the UrQMD is the input of hydrodynamic program. During hydrodynamic simulation in the equilibrium stage of collision, we model perturbations which are caused by the interaction of jets with the medium. Test of the energy loss $d E / d x$ algorithm was done on the ellipsoidal flow case. More details are in Ref. [5]. The A+A interaction simulations are major tests. Thus we performed simulation $\mathrm{Au}+\mathrm{Au}$ at $200 \mathrm{GeV} / \mathrm{c}$ and $\mathrm{Pb}+\mathrm{Pb}$ at $2.76 \mathrm{TeV} / \mathrm{c}$. For each simulation, we assume impact factor $b=3.5 \mathrm{fm}$. Our results present a qualitative analysis of energy density distribution for the fluctuations contribution assessment coming from the initial conditions and the modifications impact of jets energy deposition. Figure 1 illustrates energy density profiles in four scenarios of $\mathrm{Au}+\mathrm{Au}$ at $200 \mathrm{GeV} / \mathrm{c}$ simulations: initial condition from UrQMD averaging over 10 events and 500 events and with or without jets. Figure 1 shows the influence of initial state fluctuation on energy density shape. Figure 1 presents also energy density distributions which are perturbed by energetic parton with the $T=170 \mathrm{MeV}$ traveling through the matter at the speed of light. Results of the simulation with or without jets are consistent. The difference is only in the area of the jets propagation. On the bottom panels of the Figure 1 is a cross-section 
of the energy density (initial conditions averaging over 500 events) in the $x y$ plane where $z=0$. The energy disposition left by the jets is visible in the energy density profile. It is able to observe the formation of the Mach cone in the medium.

We performed the other four simulations of $\mathrm{Pb}+\mathrm{Pb}$ at $2.76 \mathrm{TeV}$ interactions in various scenarios. There can be visible initial state fluctuation contribution in the energy profiles in two scenarios: initial condition from UrQMD averaging over 10 events and 500 events and two variants of simulation without jet and with the jet. In such energy density magnitude, the relative energy deposits of the jet are smaller than in the previous tests (Figure 2).
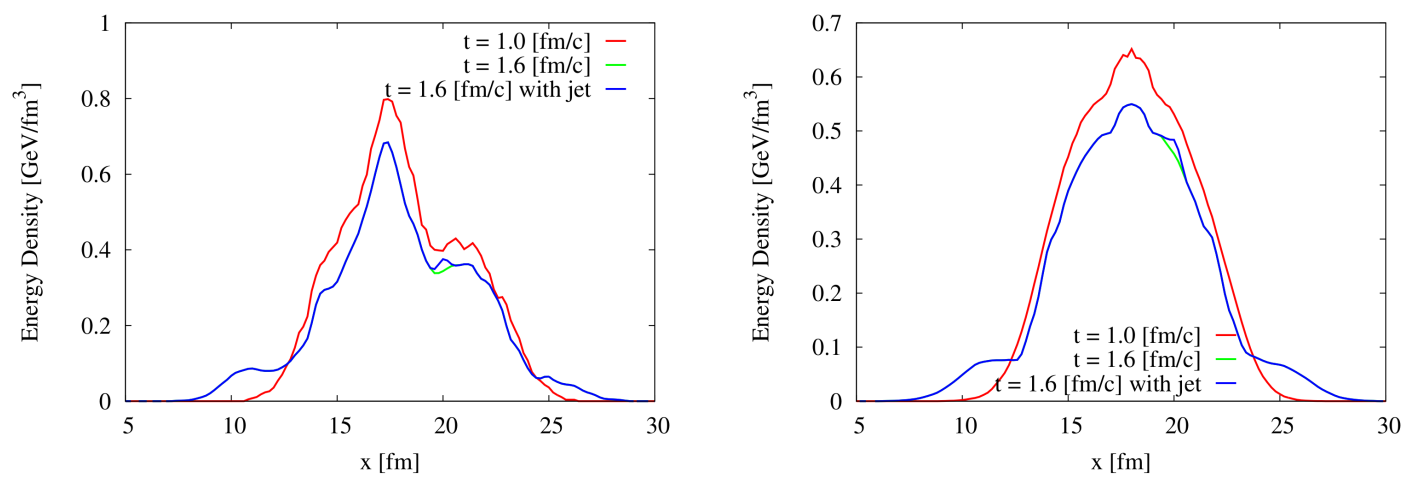

Figure 2. Energy density projection in $1 \mathrm{D}(y=z=0)$ of $\mathrm{Pb}+\mathrm{Pb}$ at $2.76 \mathrm{TeV}$. UrQMD initial conditions red line. Hydrodynamic simulations with jet - blue lines, without jet - green lines. Initial condition from UrQMD averaging over 10 events (left panel) and 500 events (right panel).

\section{Discussion}

In the performed hydrodynamic simulation, expanding QGP fluid shows strong influence of energy deposited by the jets and of initial state fluctuation. These results confirm the hypothesis of energy changes in distributions by the discussed physical effects. Those changes affect the flow anisotropy and can be compared with the results of experiments. Therefore, we are working on implementation freeze-out procedures based on Cooper-Frye formula and existing hadron freeze-out generator THERMINATOR2.

Author Contributions: conceptualization, M.S.; methodology, M.S.; software, P.G., P.M. and D.S.; validation, M.S., J.P.-S.; formal analysis, M.S., P.G. and P.M.; investigation, M.S.; resources, M.S.; data curation, M.S.; writing—original draft preparation, M.S.; writing—review and editing, M.S.; visualization, M.S.; supervision, M.S.; project administration, M.S.; funding acquisition, M.S.

Funding: This research received no external funding.

Acknowledgments: The authors thank P. Magierski for the financial support of this research from the statutory found of Nuclear Physics Division, Faculty of Physics, Warsaw University of Technology and V. Begun for useful discussions.

Conflicts of Interest: The authors declare no conflict of interest. The founding sponsors had no role in the design of the study; in the collection, analyses, or interpretation of data; in the writing of the manuscript, or in the decision to publish the results.

\section{References}

1. Shu, C.W. High order finite difference and finite volume weno schemes anddiscontinuous galerkin methods for cfd. Int. J. Comput. Fluid Dyn. 2001, 17, 107-118.

2. Casalderrey-Solana, J.; Gulhan, D.C.; Milhano, J.G.; Pablos, D.; Rajagopal, K. Comments on Entanglement Negativity in Holographic Field Theories. J. High Energy Phys. 2014, doi:10.1007/JHEP10(2014)060. 
3. Sikorski, J.; Cygert, S.; Porter-Sobieraj, J.; Słodkowski, M.; Krzyżanowski, P.; Książek, N.; Duda, P. Relativistic hydrodynamics on graphics processing units. J. Phys. Conf. Ser. 2014, 509, 012059, doi:10.1088/1742-6596/509/1/012059.

4. Bass, S.A. Microscopic models for ultrarelativistic heavy ion collisions. Prog. Part. Nucl. Phys. 1998, 41, 255, doi:10.1016/S0146-6410(98)00058-1.

5. M. Słodkowski, P. Marcinkowski, P. Gawryszewski, D. Kikoła and J. Porter-Sobieraj, Modeling of modifications induced by jets in the relativistic bulk nuclear matter, J. Phys. Conf. Ser. 2018, 1085, 052001, doi:10.1088/1742-6596/1085/5/052001

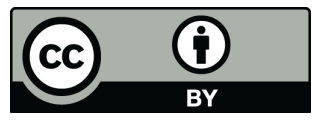

(C) 2019 by the authors. Licensee MDPI, Basel, Switzerland. This article is an open access article distributed under the terms and conditions of the Creative Commons Attribution (CC BY) license (http:/ / creativecommons.org/licenses/by/4.0/). 